\title{
Cetoacidosis diabética como forma de presentación de la diabetes gestacional en una embarazada de 25 semanas
}

\author{
Simone Pohlhammer I. ${ }^{1}$, Jorge Carvajal C. ${ }^{2}$, PhD. \\ ${ }^{1}$ Programa de Obstetricia y Ginecología, ${ }^{2}$ Unidad de Medicina Materno Fetal, División de Obstetricia y Ginecología. \\ Facultad de Medicina, Pontificia Universidad Católica de Chile.
}

\section{RESUMEN}

La cetoacidosis diabética (CAD) es infrecuente en paciente diabéticas gestacionales, sin embargo es una condición grave que tiene consecuencias maternas y fetales serias. Reportamos el caso de una embarazada, sin antecedentes mórbidos previos, con una glicemia de inicio de control prenatal normal, que debuta con CAD a las 25 semanas de embarazo. Recomendamos mantener un índice de sospecha elevado y solicitar una evaluación de glicemia a toda paciente embarazada que acude al servicio de urgencia con cuadro de náuseas y vómitos y/o deshidratación, aunque no tenga el diagnóstico de diabetes.

\section{PALABRAS CLAVE: Diabetes mellitus gestacional, cetoacidosis diabética}

\section{SUMMARY}

Diabetic ketoacidosis (DKA) is a rare, but life-threatening condition among women with gestational diabetes mellitus (GDM). We report the case of a pregnant woman without significant past medical history and with a normal prenatal fasting glucose level, that present with DKA at 25 weeks gestation. We recommend suspecting this condition and request glycaemia levels on any pregnant woman presenting to emergency room because of nausea, vomiting and dehydration, even in the absence of prior diagnosis of diabetes.

\section{KEY WORDS: Gestational diabetes mellitus, diabetic ketoacidosis}

\section{INTRODUCCIÓN}

Diabetes mellitus gestacional (DMG) es una condición clínica en la cual se reconoce por primera vez durante el embarazo cualquier grado de intolerancia a la glucosa (1). Se presenta en aproximadamente un 1 a $14 \%$ de todos los embarazos (2).

La cetoacidosis diabética (CAD) es una de las complicaciones agudas más serias de la diabetes mellitus (DM), correspondiendo a un estado de falta absoluta o relativa de insulina. Su incidencia en pacientes no embarazadas es de 4,6 a 8 episodios por 1.000 pacientes anualmente (3). Se describe una incidencia de $1,73 \%$ durante el embarazo para paciente con diabetes pregestacional usuarias de insulina (4). Se estima como muy raro un episodio de CAD en una paciente con DMG A1 de la clasificación de White.

Los casos clínicos reportados en la literatura de CAD durante el embarazo generalmente son de pacientes con DM tipo 1 no diagnosticadas (5), o pacientes con DMG en las cuales existe un factor de estrés gatillante como por ejemplo un cuadro de sepsis (6), uso de altas dosis de corticoides o uso de agonistas $B$-adrenérgicos para el manejo del parto prematuro (7).

El objetivo de esta comunicación es reportar el caso de una embarazada, que sin un factor gatillante debuta con CAD a las 25 semanas de embarazo. 


\section{Caso clínico}

Mujer de 31 años, primigesta, sin antecedentes mórbidos previos. Sin historia familiar de diabetes, embarazo bien controlado, que consulta en el servicio de urgencia de la maternidad del Hospital Clínico de la Universidad Católica con 25+4 semanas de gestación, con cuadro de una semana de evolución caracterizado por vómitos abundantes y dolor abdominal difuso (EVA 7/10) de carácter urente. No refiere fiebre, diarrea, síntomas urinarios, ni dinámica uterina. Al momento de consultar la paciente se encontraba en buenas condiciones con exámenes de laboratorio normales (amilasa sérica: $446 \mathrm{U} / \mathrm{L}$ ). Se formuló el diagnóstico de gastroenteritis aguda, y se optó por manejo sintomático ambulatorio. La paciente evoluciona con progresión de los síntomas y consulta nuevamente a las 48 horas, presentando compromiso del estado general y deshidratación.

En su segunda consulta, la paciente se encuentra lúcida orientada, con temperatura axilar de $36,5^{\circ} \mathrm{C}$, presión arterial $138 / 75 \mathrm{mmHg}$, frecuencia cardiaca 124 latidos/minuto, saturación 97\% con oxígeno ambiental, mucosas deshidratadas y labios secos. Examen cardiopulmonar normal, abdomen blando, depresible, sin signos de irritación peritoneal. Ecografía obstétrica: feto único en cefálica, latidos cardiofetales 142 por minuto, líquido amniótico normal y placenta normoinserta.

Exámenes de laboratorio:

- Hemograma: $\mathrm{Hb} 14,8 \mathrm{~g} / \mathrm{dL}$, Hematocrito $44 \%$, Leucocitos 16.400 (segmentados $85,8 \%$, linfocitos $8,1 \%)$.

- Bioquímica: PCR 0,9 mg/dL, creatinina $0,74 \mathrm{mg} /$ $\mathrm{dL}, \mathrm{Na}+138 \mathrm{mEq} / \mathrm{L}, \mathrm{K}+$ 4,8 mEq/L, Cl- 92,6 mEq/L, amilasa $1.296 \mathrm{U} / \mathrm{L}$, lipasa 22,8 U/L, SGOT $14 \mathrm{U} / \mathrm{L}$, SGPT $11 \mathrm{U} / \mathrm{L}$, bilirrubina total $0,35 \mathrm{mg} / \mathrm{dL}$, bilirrubina directa $0,14 \mathrm{mg} / \mathrm{dL}$, tiempo de protrombina 12,2 segundos (83\%, INR 1,1).

- Sedimento de orina: bacterias +, leucocitos $5 \times \mu \mathrm{L}$, eritrocitos $10 \times \mu \mathrm{L}$.

- Urocultivo negativo a las 24 horas de incubación.

Considerando el cuadro clínico de vómitos abundantes y amilasa sérica elevada la paciente ingresa con diagnóstico inicial de pancreatitis aguda y se maneja con régimen cero, hidratación intravenosa y analgesia. Evoluciona 12 horas luego del ingreso, con dificultad respiratoria, taquipnea y compromiso del estado general; se solicitan nuevos exámenes de urgencia destacando glicemia 431 $\mathrm{mg} / \mathrm{dL}$.

Con sospecha de cetoacidosis diabética (CAD) se solicitan gases en sangre venosa, que muestran $\mathrm{pH} 7.13$, bicarbonato $8 \mathrm{mEq} / \mathrm{L}$ y exceso de base $-19,3 \mathrm{mEq} / \mathrm{L}$, con cetonemia cualitativa (++), confirmándose el diagnóstico de CAD. Se traslada a Unidad de Cuidados Intermedios para manejo, con hidratación abundante, bomba de insulina, manejo de alteraciones electrolíticas y oxigenoterapia.
La embarazada evoluciona favorablemente con resolución del cuadro. Se traslada a sala al cuarto día de evolución. Es dada de alta a los 7 días, con tratamiento insulínico (NPH $12 \mathrm{U}$ predesayuno y 6 U noche + insulina cristalina según necesidad).

Mantiene controles en la unidad de alto riesgo obstétrico con vigilancia fetal y materna dentro de rango normal. Se decide inducción a las 38 semanas de acuerdo a la norma de la unidad para el manejo de pacientes con diabetes insulino requirente. El parto se resuelve por cesárea debido a estado fetal no tranquilizador (monitorización intraparto categoría 2, persistente). Se obtiene un recién nacido de sexo masculino, peso $2.930 \mathrm{~g}$, talla $49 \mathrm{~cm}, A p-$ gar 8-9, no se midió gases de cordón umbilical.

\section{DISCUSIÓN}

Presentamos el caso de una embarazada aparentemente sana sin antecedentes familiares de diabetes y con glicemia de ayuno de su primer control prenatal normal $(87 \mathrm{mg} / \mathrm{dl})$, que debuta con una cetoacidosis diabética a las $25+4$ semanas de embarazo. El cuadro clínico simuló inicialmente una gastroenteritis aguda y luego se postuló una pancreatitis. Solo la alta sospecha diagnóstica permitió el diagnóstico correcto de CAD. Si bien hubo demora en realizar el diagnóstico de CAD, debido a la alteración de la amilasa sérica que llevó al diagnóstico de ingreso de una pancreatitis aguda, el resultado materno y perinatal fueron favorables.

La cetoacidosis diabética (CAD) es una de las complicaciones agudas más serias de la diabetes. El embarazo predispone al desarrollo de cetoacidosis diabética mediante distintos mecanismos: 1 . Durante el ayuno, el uso aumentado de glucosa (extracción fetal) lleva a un metabolismo catabólico, caracterizado por hipoinsulinemia, hipoglicemia, hipercetonemia y mayor catabolismo proteico. 2. La alcalosis respiratoria relativa que existe durante el embarazo (aumento de la ventilación alveolar) genera un estado ácido-base compensatorio, aumentando la excreción de bicarbonato a nivel renal; esto se traduce en una capacidad de taponamiento ácido-base deficiente, lo cual se hace especialmente importante en la presencia de exceso de ácido como ocurre en la cetonemia. 3. La producción de hormonas diabetogénicas en el embarazo, como el lactógeno placentario, el cortisol y la prolactina predisponen a las pacientes a la cetoacidosis (8). Sin embargo, la CAD es una complicación extremadamente rara de las pacientes con DMG A1, según la clasificación de White (12).

Encontramos 27 reportes de CAD durante el embarazo, 17 corresponden a Diabetes Mellitus Pregestacional (DMPG), 6 corresponden a DMG con un factor descompensante identificable. Solo encontramos 4 casos de CAD similares al nuestro, en mujeres sin historia previa diabetes y sin un 
Tabla I

RESUMEN DE LOS ARTÍCULOS QUE REPORTAN CETOACIDOSIS DIABÉTICAS EN MUJERES EMBARAZADAS SIN DIAGNÓSTICO DE DIABETES Y SIN FACTOR DESENCADENANTE

\begin{tabular}{lcccccccc}
\hline $\begin{array}{c}\text { Raza } \\
\text { paciente }\end{array}$ & $\begin{array}{c}\text { Edad } \\
\text { materna }\end{array}$ & $\begin{array}{c}\text { Edad } \\
\text { gestacional }\end{array}$ & Paridad & $\begin{array}{c}\text { Antecedentes } \\
\text { mórbidos }\end{array}$ & $\begin{array}{c}\text { Control } \\
\text { prenatal }\end{array}$ & $\begin{array}{c}\text { Tamizaje } \\
\text { de diabetes }\end{array}$ & $\begin{array}{c}\text { Resultado } \\
\text { obstétrico }\end{array}$ & Referencia \\
\hline Asiática & 23 años & 36 semanas & 1 & No & Si & Si & $\begin{array}{c}\text { Parto por } \\
\text { Vacum * }\end{array}$ & $(8)$ \\
Negra & 34 años & 20 semanas & 2 & No & Si & Si & Óbito & $(9)$ \\
Africana & 25 años & 32 semanas & 0 & No & NC & Si & PV, $4060 \mathrm{~g}$ & $(10)$ \\
Hispánica & 33 años & 33 semanas & 1 & NC & NC & NC & Óbito & $(11)$ \\
\hline
\end{tabular}

PV: parto vaginal. NC: no consignado. *: no reporta el peso del recién nacido.

factor desencadenante de la CAD (Tabla I). Como podemos observar, los otros casos reportados son de pacientes que cursaban embarazos entre 20 y 36 semanas y todas tenían tamizaje de diabetes gestacional negativo (glicemia de ayuno en el primer trimestre). El resultado obstétrico observado es variable, sin embargo, igualmente a lo reportado en la literatura respecto de CAD durante el embarazo, la tasa de mortalidad fetal es elevada (13).

La mayoría de las veces las pacientes con CAD se presentan al servicio de urgencia con un cuadro de náuseas y vómitos asociados a deshidratación, que fácilmente podemos confundir con hiperémesis gravídica, gastroenteritis aguda o incluso pancreatitis. Como de costumbre, la anamnesis detallada, un buen examen físico y la acuciosa disquisición diagnóstica, apoyada en exámenes de laboratorio permitirá un correcto diagnóstico y manejo de la enfermedad.

La CAD durante el embarazo es una condición rara, incluso en DMG y menos en embarazadas no conocidas como diabéticas; sin embargo, representa una emergencia potencialmente letal para la madre y el feto. Recomendamos tener en mente este cuadro en embarazadas que consultan por vómitos, dolor abdominal y deshidratación, solicitando de modo rutinario una glicemia o hemoglucotest en el servicio de urgencia.

\section{REFERENCIAS}

1. Metzger BE, Coustan DR. Proceedings of the Fourth International Workshop Conference on Gestational Diabetes Mellitus. Diabetes Care 1998;21(Suppl. 2):B1-B167.
2. American Diabetes Association. Gestational Diabetes Mellitus. Diabetes Care 2004;27:s88-s90.

3. Kitabchi AE, Umpierrez GE, Murphy MB, Barrett EJ, Kreisberg RA, Malone JI, Wall BM. Management of hyperglycemic crises in patients with diabetes. Diabetes Care, 2001; 24:131-53.

4. Parker JA, Conway DL. Diabetic ketoacidosis in pregnancy. Obstet Gynecol Clin N Am 2007;34:533-43.

5. Sills IN, Rapaport R. New-onset IDDM presenting with diabetic ketoacidosis in a pregnant adolescent. Diabetes Care 1994;17:904-5.

6. Maislos M, Harman-Bohem I, Weitzman S. Diabetic ketoacidosis: a rare complication of gestational diabetes. Diabetes Care 1992;15:968-70.

7. Bedalov A, Balasubramanyam A. Glucocorticoid-induced ketoacidosis in gestational diabetes: sequela of the acute treatment of preterm labor: a case report. Diabetes Care 1997;20:922-4.

8. O'Shaughnessy MJ, Beingesser KR, Khieu WU. Diabetic ketoacidosis in pregnancy with a recent normal screening test. Western J Med 1999;170:115-8.

9. Abourizk NN. Diabetic ketoacidosis in pregnancy: another atypical case. Diabetes Care 1993;16:661-2.

10. Pitteloud N, Binz K, Caulfield A, Philippe J. Ketoacidosis during gestational diabetes. Diabetes Care 1998;21:1031-2.

11. Pinto ME, Villena JE. Diabetic ketoacidosis during gestational diabetes. A case report. Diabetes Res Clin Pract 2011;93:e92-4.

12. White P. Pregnancy complicating diabetes. Am J Med 1949;7:609-16.

13. Schneider M, Umpierrez G, Ramsey R, Mabie W, Bennett K. Pregnancy complicated by diabetic ketoacidosis: maternal and fetal outcomes. Diabetes Care 2003;26:958-9. 